\title{
A Review: The hurdle technology- Self-preservation technology in cosmetics
}

\author{
Kshitija Ballamwar*, Smt. Dr. Sangeeta Sahasrabuddhe and Komal Chafle \\ Department of Cosmetic Technology, Lady Amritbai Daga College for Women, Seminary \\ Hills, Nagpur -440006, Maharashtra, India.
}

DOI: 10.29322/IJSRP.10.08.2020.p104103

http://dx.doi.org/10.29322/IJSRP.10.08.2020.p104103

\begin{abstract}
Preservatives are added to product for basic reasons; that are to prevent microbial spoilage and thus to prolong the quantity of your time of the merchandise; and to protect the merchandise itself and customer from a possible infection. Although chemical preservative prevents microbial growth, and enhance their time period, now a days their safety is questioned by a growing segment of consumer. Therefore, there is a considerable interest within the event of preservative free or self-preservative cosmetic. In this paper, traditional/chemical preservative are replaced by other cosmetic ingredients with antimicrobial properties. And this is often commonly referred to as hurdle technology. "Hurdle technology", a technology that has been used for the limit of product protection inside the chow conscientiousness since 1970s, has besides been functional for the assembly of selfpreservative cosmetic. 'Hurdle technology' could even be a term want to describe the intelligent combination of various preservative factors or hurdle to deteriorate the expansion of microorganism. faithfulness to existing good manufacturing practice, appropriate packaging, delicate careful selection of the form of the emulsion, low water activity and low or high $\mathrm{pH}$ values are significant variables for the control of microbial growth in superficial formulation. This review article describes the appliance of the essential principles of 'hurdle technology' within the assembly of self- preservative cosmetic. Multifunctional antimicrobial ingredients and plant derived essential oils that are used as alternative or natural preservative.
\end{abstract}

Keywords: Hurdle technology, natural preservative, self-preservative, multifunctional antimicrobial ingredients.

\section{Introduction:}

The microbial safety of cosmetics is usually of particular importance in industry, since microbial route can damage the merchandise, damage the skin or inherit contact with damaged skin and pathogens, which may endanger the health of the buyer and spread the infection. With exception of pure oily preparation like petrolatum, body oils or lipsticks, the rich composition of recent cosmetic compositions alongside the aqueous medium is right soil for the propagation of microorganism. Preservatives are antimicrobial chemicals added to cosmetics to protect them from microbial disorders caused by raw materials, production, and consumers.

Paraben, the foremost widely used preservative in the world, has negligible estrogenic properties [1]. In December 2005, the security assessment of parabens was resumed after consideration of the cosmetic ingredients. Because there is a suspicion of a connection between the presence of parabens in breast issue and breast carcinoma [2,3]. In recent years, there has been a growing interest within the development of preservative-free cosmetic formulations. Preservative-free aqueous formulation are often made microbiologically stable by sterile production and appropriate packaging. However, this approach might not work for the foremost aqueous cosmetics packaged in multiple-use containers. It is to be remarked that the common definition for preservative-free means the merchandise does not contain substances that are classified as preservatives consistent with the cosmetic legislation. Therefore, the term 'Hurdle technology' (self-preservative) is more appropriate than preservative-free. The principles of 'Hurdle technology' also are applied for the assembly of self-preserving formulations.

The goal is to dam the expansion of microorganisms by fixing in their path various impediments that ought to each reduce the microorganism number but not kill the whole population. Each impediment should permit a diminished surviving population so as that because the quantity of impediments grows the number of survivors is going to be decreased and eventually reach zero. Number of the organisms may overcome the primary hurdle; of these that survived, some may overcome the second then forth until none survive the last hurdle [9]. This review article briefly reviews the techniques used for the assembly of non-classically preserved cosmetics following the concept of 'hurdle technology'. Multifunctional ingredients, plant-derived essential oils with antimicrobial properties that are used as alternative or natural preservatives.

$>$ The principles of the self-preserving technology Hurdle are presented below; 
1. Good manufacturing practice (GMP)

2. Appropriate packaging

3. Water activity

4. $\mathrm{pH}$ control

5. Multifunctional antimicrobial ingredients.

\section{Types of micro-organism:}

There is no right preservative or wrong preservative for every situation as there is no right or wrong stabilizer for every formula. The choice of ingredient that is used is, even today, largely empirical. Because when preservation is mentioned microorganisms come to mind, those that are found in cosmetics as contaminants are gone over in detail.

2.1 Fungus: A fungus is any member of the group of eukaryotic organisms that has microorganisms like yeast and molds.

Molds: -

Molds are filamentous fungi and are widely distributed throughout the earth in soil and water, and as parasites in plants and animals. They are unicellular and multicellular fungi. Their average size is about 30 micron in diameter. Molds grow best at room temperature. They require moisture and darkness to grow. The $\mathrm{pH}$ must be between 2 and 5.5 , but the only $\mathrm{pH}$ for them is 4.5 to 5.5. They go to not grow in an alkaline or neutral environment. [36]

The followings are probably the most frequent molds found in cosmetics: Examples are
a) Penicillium
b) Aspergillus
c) Rhizopus
d) Mucor Mucedo.

Yeast: -

Yeast are unicellular organisms containing no chlorophyll, but they may contain pigments ( yellow, pink, red, green, or black) . They are doing not form hyphae, are avoid or spherical in shape, and average about 5 micron in diameter, but they are going to be anywhere from 5 to 30 micron in length. Most yeasts will grow best on any medium containing fermentable sugar, will grow over a $\mathrm{pH}$ range of 2.2 to 8 (although most yeast prefer acid conditions), and usually grow best at room temperature. They usually require an abundant amount of oxygen to grow. The next are probably the most frequent yeast found in cosmetics[36].

a) Saccharomyces

b) Cryptococcus

c) Candida

d) Zygosaccharomyces.

\subsection{Bacteria: -}

The bacteria are much more difficult to generalize about than either molds or the yeasts. Most bacteria are quite small, around 0.5 to 3 microns, bacteria are single celled organisms that reproduce by binary fission, are affected by osmotic pressure and surface tension, and usually killed by high temperature.

some bacteria those are found in the environment are beneficial as well as harmful. The $\mathrm{pH}$ required to grow that between 7.2 to 7.6. examples are:[36]

a) Bacillus subtilis

b) Staphylococcus albus

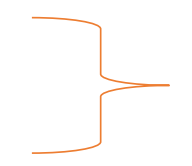
these are harmful bacteria

c) Escherichia coli

d) Lactobacillus

c) Bifidobacterium

f) Monococcus. 
Following table illustrates the types of microorganism grow in water and oil.

Table 1:- Illustrating the types of microorganism grow in water and oil.

\begin{tabular}{|c|c|}
\hline Microbes grow in water & Microbes in oil \\
\hline 1) Escherichia coli & - $\quad$ Pseudomonas \\
\hline 2) Vibrio & - $\quad$ Bacillus subtilis \\
\hline 3) Salmonella & - $\quad$ Corynebacterium sp. \\
\hline 4) Cyanobacteria & - $\quad$ Micrococcus roseus \\
\hline
\end{tabular}

\section{Mechanism of preservative:}

Preservatives counter the microbial growth in many ways. Some lead to cell wall lysis and leakage of the cell wall (e.g., phenols, organomercurials) or irreversible crosslinking at the cell wall (e.g., glutaraldehyde), while some interfere with the integrity of the plasma membrane (e.g., chelators such as EDTA, quaternary ammonium compounds). Weak carboxylic acid and alcoholic preservatives interfere with active transport mechanisms. For example, parabens and benzoic acid inhibit folic acid synthesis. Unfortunately, microorganisms become resistant to antimicrobial preservatives in multiple ways. These include one or more of the following mechanisms: (a) efflux transport mechanisms, (b) enzymatic inactivation of the antimicrobial preservative, or (c) alternation of the organism's own metabolic pathways. Some species of bacteria develop resistance to antimicrobial preservatives through genetic mutation, transduction conjugation, transformation, or transposition. These types of resistance make antimicrobial testing a critical task (29) .

\section{Needs of preservation and factors:-}

1. The use of preservative in cosmetics is essential to prevent alterations caused by microorganisms and contamination during formulation, shipment, storage or consumer use.

2. Antioxidants can also be used to protect alteration caused by exposure to oxygen.

\section{Factor affecting the action of preservatives in cosmetics:}

Fig 1:- Illustrating factors affecting the action of preservative in cosmetics 

- Concentration - more concentrated the preservative, more effective it will be $\&$ wide range of its activity .

- $\mathrm{pH}$ - microorganisms of one sort or another are capable of growing between $\mathrm{pH} 2-11$, many preservatives are $\mathrm{pH}-$ dependent.

- Surfactants - certain cationic surfactant have strong anti-microbial properties when used in combination with antiseptics. soaps \& anionics exerts mild antimicrobial properties at high conc. Non-ionics surfactants inactivate the preservatives $\&$ and also serve as source of energy for bacteria.

4)

-Solubility relationships - more soluble derivatives shows a lower inhibitory capacity.

-Partition coefficient- preservative molecules will distribute themselves between the aqueous phase, oil phase, surfactant micelles by solubilisation, and solutes by competitive displacement of water. thus, the available preservative concentrations in each of these phases will be reduced.

\section{5.material and method:}

The principles of the self-preserving technology i.e. Hurdle technology are presented below, in fig no.2

Fig 2: - Illustrating various factors of hurdle technology.

\section{Good manufacturing practices}

\subsection{Good manufacturing practice:}

Good manufacturing practices standards need to be adhered strictly during the assembly of cosmetic products when either traditional or alternative preservatives are used. Preparation of the cosmetic product under strictly aseptic condition should hinder the entry of microorganisms. Water filtration and radiation system, positive pressure, microbial testing of raw materials, disinfection of the equipment and properly trained and dressed personnel can significantly reduce the danger of contamination [910]. Thus, the utilization of strictly aseptic conditions during the assembly of cosmetic is proposed, especially when a selfpreserving system is employed within the formulations.

\subsection{Packaging and product preservation :}

If the use of preservative-free or self-preserving formulas are to be realize, then the application of the better protective cosmetic packaging must be considered a hurdle. Inclusive in this concept is the use of delivery devices which do not allow contamination to occur during use. Such as for example flip-caps for shampoos and pump-tops for lotions are example of containers that help reduce contamination during in use condition. While single-use container is an obvious solution to the problem, other more 
imaginative solutions are being developed [9-10]. In recent year such type of containers are used to preserved the product without preservative in container that can dispense product without contamination.

\subsection{Water activity and pH control}

As microorganisms require water for growth, formulations that limit the availability of water for microorganisms help control microbial growth and become one of the hurdles placed in the organism's path. The water activity (aw) describes the quantity of biologically available water within cosmetic formulations and is decided by comparing the vapor pressure of the formula containing water with the vapour pressure of pure water. Water activity may be reduced by the use of water binding substances, such as salts, polyols, protein hydrolysates, amino acids and hydrocolloids [11]. Different classes of microorganisms have different tolerance to low tide activity; bacteria generally have higher water requirements than yeasts, and yeasts higher requirements than molds [12]. Gram-negative bacteria show more susceptibility to low water activity (aw) values than grampositive. Sorbitol and glycerol, in concentrations of around $20 \% \mathrm{w} / \mathrm{w}$, are most ordinarily used to reduce water activity. However, high concentrations of polyols tend to offer the product a sticky feeling [13]. Recently, a glycerylpolyacrylate gel consisting of water, sodium polyacrylate and polyols like glycerin and ethoxydiglycol has been described for the successful preservation of oil in water $(\mathrm{O} / \mathrm{W})$ and aqueous formulations [14]. This new family of transparent, highly viscous hydrogels absorbs water from its surroundings and thus deprives microorganisms of the free water necessary for their survival. Furthermore, the hydrogels are nontoxic, non-irritant and exhibit high skin moisturizing properties[16].

\subsection{Multifunctional antimicrobial ingredients:}

According to European regulation, the only permitted preservatives are those that are listed in annex four of the $7^{\text {th }}$ amendment of the cosmetic directive. However, many cosmetic ingredients, such as alcohol, essential oil, surfactants, fatty acid, antioxidants, biomimetic phospholipid, chelating agents, have antimicrobial property. These materials which are used for their beneficial effect on the skin and may coincidentally contribute to the preservation of the formulation are not listed as preservatives of the formulation in annex four. By a careful selection of these ingredients, it is possible to decrease or to eliminate the use of traditional/chemical preservatives and to formulate cosmetics with improved cosmetic properties. Below are listed some alternative preservatives[16].

1) Surfactants

2) Fatty acid and ester

3) Biomimetic phospholipids

4) Antioxidants as preservatives

5) Aroma chemicals as preservatives

6) Chelating agents as preservatives potentiators:

7) Fragrance ingredients

\section{Surfactants in self-preserving formulas:}

Surfactants are classified as anionic, cationic or non-ionic, when present in aqueous solution at concentrations above their critical micelle concentration (CMC) surfactants can form micelles. Preservatives absorbed or enclosed in micelles have decreased preservative efficacy due to a reduction in preservative concentration. Surfactants on the other hand may help solubilize preservatives thereby making them more effective[19].

Following figure illustrating the classification of surfactant.

Fig 3-classification of surfactant.

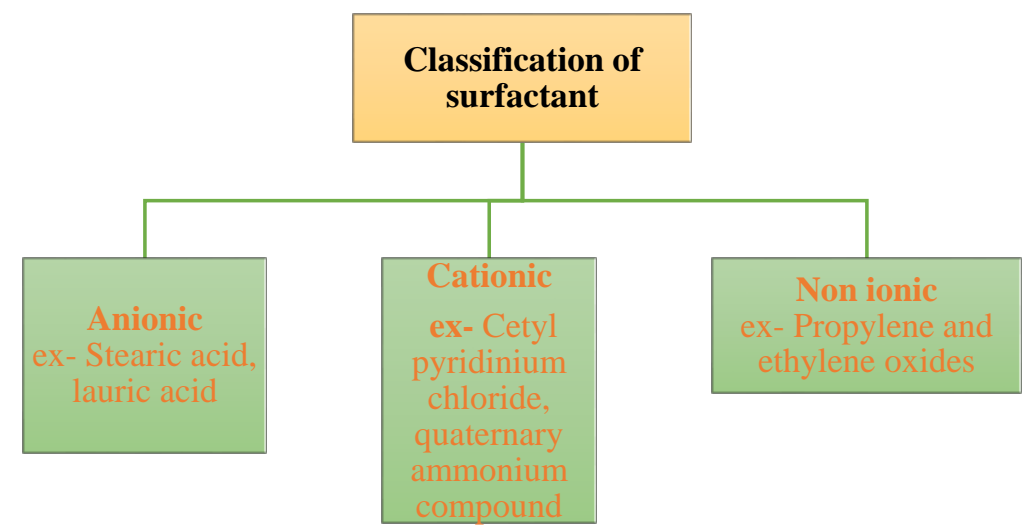




\section{a. Anionic Surfactants:-}

Anionic surfactants such as fatty acid soaps are the earliest example of an anionic compound helping preserve products. These anionic surfactants have weak antibacterial effects under alkaline conditions and generally are more active against gram positive rather than gram negative organisms. The general resistance of gram- negative organism is due to the protection afforded by their outer membrane. This protection can be lowered or reduced by the concurrent use of chelating agents[19].

\section{b. Cationic surfactants:-}

Ionize in aqueous solution to produce positively charged organic ions that are responsible for surface activity. Since 1935 when Domagt identified the antimicrobial effects of cationic surfactants, quaternary salts (e.g BAC, CPC) have been used in sanitizers, cosmetics, mouthwashes and toothpastes. Benzalkonium chloride (BAC) and cetyl pyridinium chloride (CPC) are most often used in such formulations. Their presence reduces and eliminates the need for additional preservatives. One advantage of quarts are their broad spectrum of antimicrobial activity over a range of $\mathrm{pH}$ values[19].

\section{Fatty acids and esters as multifunctional components :}

Medium chain saturated carboxylic acid acids, like heptanoic (C7), caprylic (C8), capric (C10) and dodecanoic acid (C12) and their esters with glycerine or propanediol are found to possess activity against enveloped viruses and various bacteria and fungi in vitro [19-21]. Inhibitory properties in terms of minimum inhibitory concentration (MIC) values reach a peak with $\mathrm{C} 12$ aliphatic chains and reduce rapidly at values but 8 or greater than 12 [17]. The mechanism by which the monoglycerides kill bacteria has not been completely elucidated yet, but microscope studies indicate that they disrupt cell membranes, leaving the bacterial cell intact [18-21].

\section{Biomimetic phospholipids:-}

Phospholipids are another example of emulsifiers which may have special properties. While some phospholipids (lecithin) can inactive preservatives, lipids which mimic structure and performance of phospholipids are prepared that have antimicrobial activity. This new family of phospholipids has been developed during which the arrangement of the phosphate and quaternary groupings has been reversed. Instead of interfering with preservative systems the biomimetic phospholipid displays potent antimicrobial activity without showing significant toxicity or skin irritation effects [19].

\section{Antioxidants as preservatives:-}

The primary function of phenolic antioxidants is to delay the auto-oxidation of unsaturated oils that would influence the colour and therefore the odour of the merchandise. Primary antioxidant is phenolic compound. Butylated hydroxyanisole (BHA) butylated hydroxytoluene (BHT), tertiary butylhydroquinone (TTBHQ) and propyl gallate (PG) are the foremost common phenolic utilised in cosmetics. BHA and TBHQ are the foremost effective antimicrobial of the phenolic antioxidant and are foremost active than the standard parabens. Although, better effective against gram positive organism, these phenolics are active against certain gram-negative organisms also as mold and yeasts. Propyl gallate is quite a water-soluble molecule with antioxidant and antimicrobial activities against bacteria and fungi at a degree of concentration $0.5 \%(\mathrm{w} / \mathrm{w})$ [13]. Other examples are Caffeic, coumaric and ferulic acid have also demonstrated antimicrobial activity [28-32]. The selection of antioxidant depends on the convenience of corporation, composition of the merchandise, the order of addition and packaging. Safety, compliance with governmental regulations and price should be considered. Final selection of the "hurdle" to be utilized in a preservative system depends on the precise of the formula, the manufacturing process and therefore the end use by the buyer [19].

\section{Aroma chemicals as preservatives:}

Spices and essential oils have long been wont to prevent the deterioration of cosmetic products. Because most studies for evaluating essential oils and fragrances used agar streak or disk method, true efficacy of the many extracts weren't properly evaluated. Despite these short comings the antimicrobial properties of aromatic substances were found to be useful. Aroma chemicals are multifunctional in order that their use in cosmetic products shouldn't only be supported the specific scent but also upon its inherent germicidal properties [19]. The phenol coefficient may be a measure of the bactericidal activity of a chemical compound in reference to phenol. The activity of compound is expressed because the ratio of dilution during which it kills in 10 minutes but not in 5 minutes under the required conditions. It is often determined within the absence of organic matter, or within the presence of a typical amount of added organic matter. In contrast, a phenol coefficient that but 1 means the disinfectant may be a smaller amount effective than phenol. 
Table no. 2 Essential oil and their phenol coefficient. Mention in text

\begin{tabular}{|c|c|}
\hline Essential oil & Phenol coefficient \\
\hline 1) Oil of clove & 9 \\
\hline 2) Oil of thyme & 15 \\
\hline 3) Oil of cinnamon & 9 \\
\hline 4) Oil of rose & 6 \\
\hline 5) Oil of lemon & 4 \\
\hline 6) Oil of eucalyptus & 4 \\
\hline 7) Oil of lavender & 5 \\
\hline
\end{tabular}

\section{Chelating agents as preservatives potentiators:}

The chelators, EDTA, carboxylic acid, acid and phytic acid increase the permeability of cell membranes and make them more sensitive to antimicrobial agents. additionally, chelating agents block the iron needed for microbial metabolism and growth. Thus, chelators could be important ingredients in enhancing the efficacy of antimicrobial agents for the control of gram-negative bacteria which are known to possess increased resistance to antimicrobial agents [27,28].

\section{Fragrance ingredients:-}

The chemical composition of antimicrobial fragrances isn't an excessive amount of various from antimicrobial essential oils and extracts. within the past, a fragrance mixture mainly comprised of benzyl acetate, phenethyl alcohol and Linalool which was utilized so on reduce the quantity of parabens utilized in cosmetic formulations [35]. At now, antimicrobial perfumes are commercially available, p-anisic acid (p-methoxy-benzoic acid) and levulinic acid (4oxopentanoic acid) being the foremost components of them. p-Anisic acid is found in Pimpinella anisum and other herbs and levulinic acid has been found as by-product within the assembly of diosgenin from wild yam (Dioscorea villosa) [15]. However, replacement of the chemical preservatives by fragrance ingredients won't necessarily ensure a reduction within the irritating effect of the formulation.

\section{Conclusion: -}

This new preservative system approach for product protection includes compounds (hurdle) that have multi-functional activity and packaging components which will contribute directly or indirectly to keeping the environment hostile to microorganisms. Preservative free or self-reserving formulas offer numerous advantages. Studies that on some traditional/ chemical preservatives i.e. parabens, formaldehyde releasers and isothiazolinones together, increased the desire of consumers, for 'natural' products that has led the cosmetic industry to the enhancement of latest preservation methods. The devotion to modern GMP and therefore the use of appropriate packaging together with the control of crucial factors for the expansion of microorganisms i.e. water activity (aw) and $\mathrm{pH}$ can remarkably decrease the number of traditional/chemical preservatives needed for the stableness of a cosmetic formulation. In self-preserving formulations, old preservatives are replaced by other chemical ingredients with antimicrobial properties i.e. surfactants, fatty acid, chelating agents, essential oils, etc. These materials which are used for his or her beneficial effect on the skin and coincidentally contribute to the preservation of the formulation. However, the utilization of such alternative or natural substances doesn't ensure complete elimination of adverse events, irritating effects or sensitization. The 'ideal solution' which can replace traditional/chemical preservatives and may be absolutely safe, effective and compatible for all applications has not been found yet and doubtless never will. This hurdle technology is that the ideal solution.

\section{$\underline{\text { Reference }}$}

1. Lemini, C., Jaimez, R., Avila, M.E., Franco, Y., Larrea, F. and Lemus, A.E. In vivo and in vitro estrogen bioactivities of alkyl parabens. Toxicol. Ind. Health 26, 69-79 (2003).

2. Darbre, P.D., Aljarrah, A., Miller, W.R., Coldham, N.G., Sauer, M.G. and Pore, G.S. Concentration of parabens in human breast tumors. J. Appl. Toxicol. 24, 5-13 (2004).

3. Epstein, H. Cosmetics preservation. Clin. Derm. 24, 551-552 (2006).

4. Consumer Product Safety Commission. An Update on Formaldehyde 1997 Revision, CPSC Document \# 725. Available at http://www.cpsc.gov/cpscpub/ pubs/725.html, accessed 10 October (2007). 
5. Reinhard, E., Waeber, R., Niederem, M., Mauer, T., Maly, P. and Sherer, S. Preservation of products with MCI/MI in Switzerland. Contact Derm. 45, 257-264 (2001).

6. Varvaresou, A., Tsirivas, E. and Tsaoula, E. Isothiazolinone biocides as preservatives in cosmetics. Rev. Clin. Pharmacol. Pharmacokinet. 19, 105-108 (2005).

7. Leistner, L. Basic aspects of food preservation by hurdle technology. Int. J. Food Microbiol. 55, 181- 186 (2000).

8. Sen`orans, J., Iban`ez, E. and Cifuentes, A. New trends in food preserving. Crit. Rev. Food Sci. Nutr. 43, 507-526 (2003).

9. Kabara, J.J. and Orth, D.S.(eds) Preservative-Free and Self-Preserving Cosmetics and Drugs: Principles and Practice. Marcel Dekker Inc., New York (1996).

10. Kabara, J.J. and Orth, D.S. Preservative-free and selfpreserving cosmetics and drugs. Cosmet. Toiletries 113, 51-58 (1998).

11. Franke, J. and Meyer, B. Mo“glichkeiten kosmetischer Mittel durch niedrige Wasseraktivita“t. SO *WF J. 120, 317-321 (1994).

12. Orth, D.S. Handbook of Cosmetic Microbiology, Marcel Dekker Inc., New York pp. 521-582. (1993).

13. Petersen, W. Antimicrobial ingredients for self-preserving cosmetics. Eur. Cosmet. 2/99, 28-36 (2002).

14. Lintner, K. and Genet, V. A physical method for the preservation of cosmetic products. Int. J. Cosmet. Sci. 20, 115-133 (1998).

15. Dweck, A.C. Natural preservatives. Cosmet. Toiletries 118, 45-50 (2003).

16. A. Varvaresou, S. paoageorgiou, E. tsirivas, E. Protopapa, H. Kintziou, V. kefala and c. demetzos self- preserving cosmetics.163-175(2009).

17. Ja"nichen, J. The quest for the ideal preserving system-reducing traditional preservatives in combination with Dermosoft Octiol. Eur. Cosmet. $7 / 8$, $10-16$ (2004).

18. Kabara, J.J., Swieczkowski, D.M., Conley, A.J. and Truant, J.P Dr Straetmans Formulatory, Hamburg, Germany, www.dr-straetmans.de 19. Fatty acids and derivatives as antimicrobial agents. Antimicrob. Agents Chemother. 2, 23-28 (1972).

19. Kabara, Jon J. Wachter Road, Galena, Illinois 61036 U.S.A. hurdle technology: are biocides always necessary for product protection J. Appl. Cosmetol. 17, 102-108 (July/September 1999).

20. Bergsson, G., Arnfinnsson, J., Steingrı 'mson, O '. and Thormar, H. In vitro killing of Candida albicans by fatty acids and monoglycerides. Antimicrob. Agents Chemother. 45, 3209-3212 (2001).

21. Bergsson, G., Arnfinnsson, J., Steingrı 'mson, O'. and Thormar, H. Killing of gram-positive cocci by fatty acids and monoglycerides. APMIS 109, 670-678 (2001).

22. Manning, M. and Orawski, P. Food grade esters used as personal care antimicrobials. Cosmet. Toiletries 120, 63-68 (2005).

23. Lilley, B.D. and Brewer, J. The selective antibacterial action of phenylethyl alcohol. J. Am. Pharm. Assoc. Sci. Ed. 42, 6-8 (1953).

24. Silver, S. and Wendt, L. Mechanism of action of phenethyl alcohol: breakdown of the cellular permeability barrier. J. Bacteriol. 93, 560-566 (1967).

25. Kaszowska, M. Chemical structure and biosynthesis of lipopolysaccharide - important component of the cell envelope of gram-negative bacteria. Postepy Hig. Med. Dosw. (Online) 58, 333-342 (2004).

26. Ouattara, B., Simard, R.E., Holley, R.A., Piette, G. and Be 'gin, J.-P. Antibacterial activity of selected fatty acids and essential oils against six meat spoilage organisms. Int. J. Food Microbiol. 37, 155-162 (1997).

27. Graf, E. Applications of phytic acid. J. Am. Oil Chem. Soc. 60, 1861-1867 (1983).

28. Graf, E., Empson, K.L. and Eaton, J.W. Phytic acid. J. Biol. Chem. 262, 1647-11650 (1987).

29. Graf, E., Empson, K.L. and Eaton, J.W. Phytic acid. J. Biol. Chem. 262, 1647-11650 (1987).

30. CIR. Final Report on the Safety Assesment of Propyl Gallate. CTFA Inc., New York, NY, USA (1981).

31. Eggenspeger, H. and Wilker, M. Zur multiaktiven Wirkung von Ferulasa“ure und ihren Estern in Kosmetika. SO *WF J. 122, 146-156 (1996).

32. Eggenspeger, H., Ihlbrock, D. and Bauer, P. Zur kosmetischen wirkung von Extraken aus Pflanzenmischungen. SO “WF J. 124, 2-9 (1998).

33. Branen, A.I. and Davidson, P.M. Use of antioxidants in self-preserving cosmetics and drugs. In: Preservative-Free and Self-Preserving Cosmetics and Drugs: Principles and Practice (Kabara, J.J. and Orth, D.S., eds), . Marcel Dekker Inc., New York 159-179.(1996).

34. Hinou, J.B., Harvala, C.E. and Hinou, E.B. Antimicrobial activity screening of 32 common constituents of essential oils. Pharmazie 44, 302-303 (1989).

35. Blakeway, J.M. Fragrances as preservatives. SO “FW J. 116, 357-359 (1990).

36. John Wiley and Sons, M.S. Balsam and Edward Sagrin cosmetic science and technology sec.edi.vol.3 394-398. 